\title{
Atualização do Tratamento Medicamentoso da Osteoporose
}

\section{Osteoporosis Drug Treatment Update}

\author{
Lindomar Guimarães Oliveira ${ }^{1}$ Mara Lúcia Rassi Guimarães Carneiro ${ }^{1}$ \\ Márcio Passini Gonçalves de Souza ${ }^{2}$ Caio Gonçalves de Souza ${ }^{2}$ Frederico Barra de Moraes ${ }^{1}$ \\ Fábio Lopes de Camargo ${ }^{1}$
}

${ }^{1}$ Departamento de Ortopedia e Traumatologia, Universidade Federal
de Goiás, Goiânia, GO, Brasil
2 Instituto de Ortopedia e Traumatologia, Hospital das Clínicas, Faculdade
de Medicina, Universidade de São Paulo, São Paulo, SP, Brasil

Rev Bras Ortop 2021;56(5):550-557.

\begin{abstract}
Endereço para correspondência Frederico Barra de Moraes, Departamento de Ortopedia e Traumatologia, Universidade Federal de Goiás, Rua Teresina, 30, apto 1202 - Edf. Spazio Gran Ville, Goiânia, GO 74815715, Brasil (e-mail: frederico_barra@yahoo.com.br).
\end{abstract}

\section{Resumo \\ Palavras-chave \\ - envelhecimento \\ - osteoporose/ diagnóstico \\ - osteoporose/terapia}

A população brasileira está envelhecendo, e com isso aumenta a prevalência de doenças crônico-degenerativas, dentre elas a osteoporose. O diagnóstico e tratamento da osteoporose teve avanços significativos na última década. O ortopedista e traumatologista não pode mais se deter apenas no tratamento cirúrgico da fratura osteoporótica. É extremamente importante que saibamos: 1 ) quais fatores de risco avaliar, podendo ser utilizada a ferramenta Fracture Risk Assesment Tool (FRAX, na sigla em inglês); 2) quais exames complementares solicitar, como densitometria, radiografia da coluna e bacia, exames de sangue e urina, e até mesmo biópsia óssea; 3) quais suplementos utilizar, como cálcio e magnésio, vitaminas D e K; 4) quais medicamentos prescrever, antirreabsortivos ou formadores, janelas terapêuticas e eventos adversos.

The Brazilian population is aging and the prevalence of chronic degenerative diseases, including osteoporosis, is increasing. The diagnosis and treatment of osteoporosis have made significant advances in the last decade. The orthopedist and traumatologist can no longer be detained only in the surgical treatment of osteoporotic fracture. It is extremely important that we know: 1) what risk factors to evaluate, and the Fracture Risk Assesment Tool (FRAX) can be used; 2) which complementary tests should be requested, such as densitometry, radiography of the spine and pelvis, blood and urine tests, and even bone biopsy; 3 ) which supplements to use, such as calcium and magnesium, vitamins $\mathrm{D}$ and $\mathrm{K} ; 4$ ) which medications to prescribe, antiresorptives or trainers, therapeutic windows and adverse events. recebido

03 de Fevereiro de 2020

aceito

15 de Abril de 2020
DOI https://doi.org/

$10.1055 / \mathrm{s}-0040-1714219$ ISSN 0102-3616.

\footnotetext{
(c) 2021. Sociedade Brasileira de Ortopedia e Traumatologia. All rights reserved.

This is an open access article published by Thieme under the terms of the Creative Commons Attribution-NonDerivative-NonCommercial-License, permitting copying and reproduction so long as the original work is given appropriate credit. Contents may not be used for commercial purposes, or adapted, remixed, transformed or built upon. (https://creativecommons.org/ licenses/by-nc-nd/4.0/)

Thieme Revinter Publicações Ltda., Rua do Matoso 170, Rio de Janeiro, RJ, CEP 20270-135, Brazil
} 


\section{Introdução}

Quando pretendemos tratar um paciente com osteoporose, algumas perguntas nos vêm à mente. A primeira é: por que tratar? E a resposta é porque queremos evitar a alta prevalência de complicações causadas por essa doença. Após os 50 anos de idade, aproximadamente metade das pessoas terão osteopenia ou osteoporose, sendo que desses, metade irão sofrer pelo menos uma fratura por trauma mínimo. No mundo, temos uma fratura osteoporótica a cada 3 segundos, e a tendência é que esse número aumente à medida que a população envelhece mais. ${ }^{1,2}$

Osteoporose nos Estados Unidos resulta em 1,5 milhões de fraturas por ano, a maioria em mulheres na pós-menopausa. Fraturas do quadril podem resultar em piora na qualidade de vida, perda da independência e aumento do risco de morte. Fraturas vertebrais, também associadas a risco de morte, podem resultar em dor crônica, cifose e perda da qualidade de vida., ${ }^{1,2}$

No Brasil, o número de pessoas que possuem a doença chega a 10 milhões, e os gastos com o tratamento e a assistência no Sistema Único de Saúde (SUS) são altos. Só em 2010, o SUS gastou $\sim \mathrm{R} \$ 81$ milhões para a atenção ao paciente portador de osteoporose e vítima de quedas e fraturas ${ }^{3}$ Considerando o envelhecimento populacional, e pelo fato de fraturas não serem de comunicação obrigatória, o número real brasileiro é muito maior. Freedman et $\mathrm{al}^{4}{ }^{4}$ em 2007, chamavam atenção que o ortopedista perdia a primeira oportunidade para tratar osteoporose quando atendia pacientes com fraturas do punho. O cenário pouco mudou entre os ortopedistas quando lidam com a manifestação clínica da osteoporose, que é a fratura. $\mathrm{O}$ ortopedista tem o dever de indicar tratamento ou encaminhar o paciente para tratamento, e no Brasil essa atitude ainda é pequena. ${ }^{2,3}$

Em várias cidades no Brasil, existem serviços organizados para tratar osteoporose e prevenir a refratura, tendência mundial com os chamados Fracture Liaison Services (FLSs), sendo um deles no Brasil o PREVREFRAT. ${ }^{3}$ Sempre há um profissional dedicado a este tipo de atividade, o qual pode ser o ortopedista. Com o tratamento da osteoporose, queremos evitar a alta mortalidade após as fraturas por fragilidade no primeiro ano pós-fratura (30\%), além de evitar a alta morbidade como a dependência para deambular (60\%) ou ainda a perda de alguma capacidade de realizar atividades da vida cotidiana (90\%). Tratar osteoporose diminui a mortalidade dos pacientes, e essa deve ser nossa preocupação principal. ${ }^{1-6}$

\section{Para Quem Indicar o Tratamento da Osteoporose}

A segunda pergunta é: como identificar quem precisa ser tratado? De acordo com a National Osteoporosis Foundation (NOF, na sigla em inglês), as recomendações para o tratamento da osteoporose em mulheres e homens após os 50 anos de idade são as seguintes ${ }^{1-6}$ :

1. Presença de fratura vertebral ou femoral por fragilidade (trauma mínimo);
2. Densitometria óssea com T-score $\leq-2,5$, isto é, osteoporose;

3. Pacientes com T-score entre -1,1 e -2,4 (osteopenia), e com Fracture Risk Assesment Tool (FRAX, na sigla em inglês) positivo;

Antes de instituir tratamento, o paciente deve ser avaliado para diagnóstico diferencial da osteoporose, como por exemplo: osteomalácea, mieloma múltiplo, desnutrição, doenças gastrointestinais ou renais, entre outras. Na suspeita de alguma doença que cursa com perda óssea, devem ser solicitados exames para diagnóstico de osteoporose secundária. Deve-se verificar as comorbidades existentes em tratamento. Na história clínica, deve-se buscar os fatores de risco. Os fatores de risco mais importantes são: idade, sexo feminino, etnias branca e oriental, história familiar de fratura prévia e pessoal, baixa densidade mineral óssea (DMO), uso de glicocorticóides (dose $\geq 5.0 \mathrm{mg} /$ dia de prednisona $>3$ meses), fatores ambientais, tabagismo, alcoolismo ( $\geq 3$ doses por dia), sedentarismo, baixa vitamina $\mathrm{D}$ e baixa ingestão de cálcio. Intolerância à lactose e baixa exposição solar são relevantes como risco. ${ }^{6}$

Para início do tratamento, os fatores de risco modificáveis devem ser eliminados, por exemplo: baixo peso, alcoolismo, tabagismo, sedentarismo, deficiências nutricionais, e outros de possível atuação. Mulheres têm maior incidência de osteoporose e de fraturas osteoporóticas do que os homens, porém na mortalidade pós-fratura os homens têm maior incidência. Causas de osteoporose secundária podem ser identificadas em $\sim 40$ a $60 \%$ dos homens, principalmente os portadores de fraturas osteoporóticas. As mais comuns são hipogonadismo e corticoterapia prolongada, seguido de doenças gastrointestinais, deficiência de vitamina D, alcoolismo e anticonvulsivantes. Exames de sangue e urina nos ajudam a realizar esses diagnósticos, sendo os principais: hemograma, hemoglobina glicada, hormônio tireoestimulante (TSH), creatinina, cálcio e fósforo séricos, elementos anormais de sedimento (EAS) tipo 1 e calciúria de 24 horas, paratormônio (PTH), 25-OH-Vitamina D, c-telopeptídeo (CTX-1), fosfatase alcalina e eletroforese de proteínas. ${ }^{7}$ Com esses exames, conseguimos diagnosticar $>80 \%$ das causas secundárias e estabelecer o tratamento medicamentoso com segurança. Em casos mais raros, pode ser necessária biópsia óssea, inclusive com avalição histomorfométrica.

A evolução clínica da osteoporose se assemelha entre os dois sexos na fase senil $>70$ anos de idade. Existe um novo conceito, para rápida atuação, chamado de risco iminente de fraturas, que é a possibilidade maior de refraturas que pode ocorrer no primeiro e segundo ano após a primeira fratura por fragilidade, osteoporose com presença de fatores de risco importantes e doenças preexistentes. ${ }^{8}$

Densitometria óssea é o exame padrão ouro atual para diagnóstico da osteoporose, com os resultados normais: $T$ -Score de 0 a - 1, baixa densidade mineral óssea (tendência de abolir o nome osteopenia) de - 1,1 a - 2,4, e osteoporose densitométrica $\leq-2,4$ para baixo. É considerado para leitura o sítio de menor valor no exame. Presença de fratura por fragilidade e fatores de risco diagnóstico clínico de osteoporose, e indicar tratamento. Valor de Z-Score $\leq-2$ indica 
pesquisa para investigar possível existência de fator causal para osteoporose secundária. Presença de fratura por fragilidade com outros fatores de risco é diagnóstico clínico de osteoporose independentemente do valor na densitometria, com indicação formal de tratamento. ${ }^{2-6}$

A fratura por trauma leve (queda da própria altura) ou fragilidade óssea já é indicação para o tratamento. Diversas diretrizes indicam tratamento em $\mathrm{T}$-Score $<-2.4$ e presença de fatores de risco, na ausência de fraturas. É importante o médico ter em mente os fatores de risco no momento da consulta; diversos algoritmos matemáticos foram desenvolvidos para o auxílio do diagnóstico e avaliar o risco de fratura. O algoritmo mais utilizado atualmente para cálculo do risco de fraturas em 10 anos é o FRAX, ferramenta que utiliza para cálculo fatores de risco mais importantes, como idade, sexo, alcoolismo, índice de massa corporal (IMC) e outros, como a densidade mineral óssea (DMO) do colo femoral. O FRAX Brasil pode ser buscado no site www.shef.ac.uk/FRAX e na ABRASSO https://abrasso.org.br/calculadora/calculadora. O FRAX faz predição da fratura por osteoporose com fatores de risco mesmo sem a DMO. Pode ser utilizado em homens e mulheres com osteopenia (baixa densidade mineral óssea) ou osteoporose, e é validado no Brasil. ${ }^{6-9}$

\section{Suplementos Usados Para Osteoporose}

Todos os pacientes com perda óssea, ou em potencial de risco para perda, devem ser aconselhados para o uso alimentar de cálcio e vitamina D ou suplementos. A absorção do cálcio diminui com a idade. De 30 a 50\% do cálcio ingerido pelos adultos é absorvido pelo intestino. A vitamina $\mathrm{D}$, sendo um hormônio, ativa a absorção do cálcio intestinal, sendo necessário suplementar pessoas idosas, sedentárias ou internadas. ${ }^{3-6}$

Carbonato de cálcio, o mais comum em cápsulas, contém $40 \%$ de cálcio, então para $500 \mathrm{mg}$ do elemento se necessita $1.250 \mathrm{mg}$ de carbonato, melhor aproveitado quando ingerido junto a uma refeição. O carbonato de cálcio pode provocar obstipação intestinal, sendo relatado como causa de cálculos renais. Para pacientes com gastrectomia, história de calculose e cirurgia bariátrica, são melhores indicados o trifosfato de cálcio ou o citrato de cálcio, cuja molécula tem $\sim 20 \%$ do elemento, mas com absorção em maior quantidade. Consumo de suplementos de cálcio $>2.000 \mathrm{mg}$ por dia está relacionado com aumento do risco de eventos cardiovasculares e cálculos renais, mas o cálcio ingerido da dieta é mais seguro quanto a efeitos colaterais. ${ }^{10-12}$

Evidências indicam que alimentação diária incluindo lácteos, frutas, verduras e adequada quantidade de carnes, peixes e aves, contribui para saúde óssea. ${ }^{11,12}$ A International Osteoporosis Society (IOF, na sigla em inglês) tem um programa para cálculo da ingestão de cálcio com alimentação em https://www.iofbo. ${ }^{13}$

A vitamina $D$ é um pró-hormônio sintetizado na pele quando exposta aos raios ultravioletas B (UVB) da luz solar. As fontes de vitamina D alimentares são escassas e os seres humanos dependem principalmente da estimulação cutânea pelos raios UVB solares. A vitamina $D$, armazenada na gordura subcutânea da pele, sofre transformações químicas transformando em sua forma ativa (calcitriol), com importantes funções na fisiologia ósseo-mineral, especialmente no que se refere à absorção intestinal e à homeostase do cálcio. ${ }^{6}$

Além de seu papel na absorção intestinal de cálcio, a vitamina $\mathrm{D}$ exerce importante ação na musculatura periférica e no equilíbrio, potencialmente diminuindo o risco de quedas. A deficiência de vitamina D é comum em pacientes com osteoporose e fraturas de quadril. Para dosagem da vitamina $\mathrm{D}$, deve ser solicitada a hidroxivitamina $\mathrm{D}(25[\mathrm{OH}]$ D), que é a forma circulante; o valor de corte considerado normal para adultos é de $30 \mathrm{ng} / \mathrm{ml}$. Em adultos com deficiência de vitamina D $(25[\mathrm{OH}] \mathrm{D}<20 \mathrm{ng} / \mathrm{ml})$, recomenda-se a administração de uma dose de ataque de $7.000 \mathrm{UI} /$ dia ou de $50.000 \mathrm{UI} /$ semana por 8 semanas, seguida da dose de manutenção entre 1.000 e 2.000 UI por dia. Recomenda-se dosar os níveis de vitamina D no seguimento clínico após 4 meses. ${ }^{12}$

O magnésio ajuda na estabilização do fosfato de cálcio na hidroxiapatita e sua dose diária recomendada é de $320 \mathrm{mg}$. A vitamina K2, que é encontrada nos alimentos verdes, carboxila a osteocalcina, uma proteína óssea que auxilia na mineralização óssea, além de diminuir a calcificação vascular. Os suplementos proteicos mais usados são o "whey protein" (WP, na sigla em inglês), os "branched-chain amino-acids" (BCAA, na sigla em inglês) e os colágenos hidrolisados, para ganhos de massa muscular e óssea. ${ }^{13,14}$

\section{Medicamentos Usados Para Tratamento da Osteoporose}

Do ponto de vista farmacológico, temos duas classes de medicamentos para osteoporose. Os antirreabsortivos (anticatabólicos), e os estimuladores da formação óssea (anabólicos). Os osteócitos são as células responsáveis pelo controle de todo o processo de renovação do esqueleto por perceberem as microfraturas em seus canalículos. Os antirreabsortivos atuam inibindo os osteoclastos, que são as células responsáveis por iniciar a remodelação óssea, reabsorvendo áreas de microfraturas ou de osso frágil, levando à formação das lacunas de Howship. Os formadores são aqueles que estimulam os osteoblastos a produzirem massa óssea, preenchendo essas lacunas com matriz osteóide renovada, que será posteriormente mineralizada, melhorando as propriedades físicas (deformidade sob carga) e biológicas (histomorfometria tecidual) do osso para evitar fraturas. O osso é um tecido vivo que precisa ser renovado continuamente, a uma taxa de $10 \%$ ao ano. Osso que não se renova fratura mais fácil pois perde sua viscoelasticidade. ${ }^{6}$

Os antirreabsortivos são subdivididos em: 1) Hormonais: terapia de reposição hormonal (TRH) (estrógenos e testosterona); calcitonina (hormônio tiroidiano); raloxifeno (inibidor seletivo dos receptores de estrógeno [SERMS]); 2) bisfosfonatos: não nitrogenados ou nitrogenados; alquil ou heterocíclicos; 3 ) biológicos: Denosumabe (ligante do receptor ativador do fator nuclear kappa B [RANKL, na sigla em inglês]). Os anabólicos atuam estimulando a formação óssea e por isso são estimuladores do metabolismo do osso. Atualmente, temos em uso a teriparatida, análogo do PTH. Recente lançamento nos EUA e Europa da Abaloparatida 
Tabela 1 Medicamentos aprovados pela ANVISA para tratamento da osteoporose

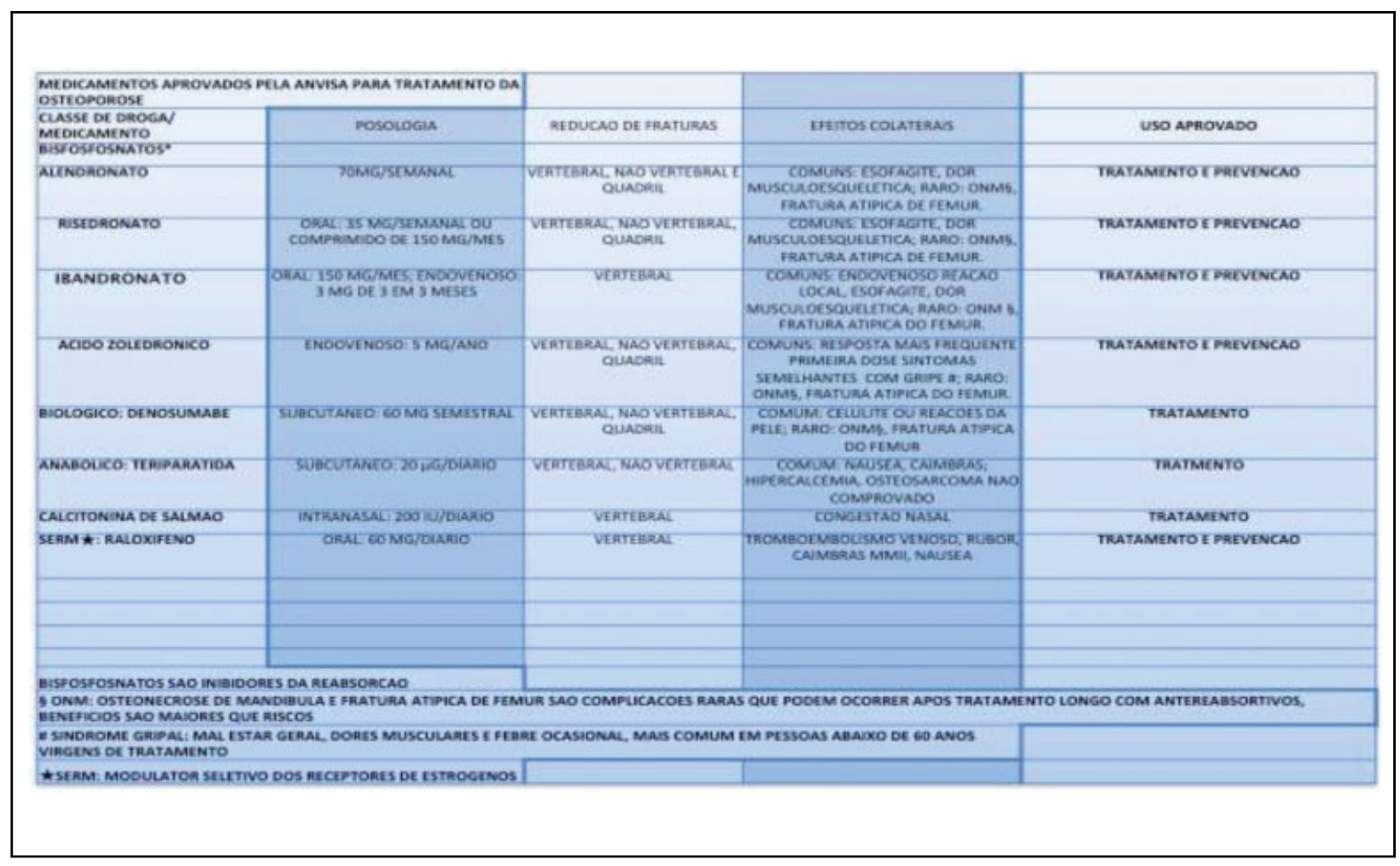

(similar do PTH), e um biológico - Romosozumabe (anticorpo monoclonal humano anti-esclerostina), aprovado nos EUA e no Japão. Ambos aguardando liberação no Brasil pela ANVISA $^{13-33}$ (- Tabela 1 ).

\section{Terapia de Reposição Hormonal}

A terapia de reposição hormonal feminina deve ser indicada pelo ginecologista em vigência dos transtornos sintomáticos da menopausa. Pode ser considerada como tratamento preventivo da osteoporose na prevenção de fraturas, quando os riscos de uso no paciente são inferiores ao benefício, e para tratar os distúrbios vasomotores da menopausa. A reposição hormonal masculina com testosterona pode ser indicada com avaliação do urologista, na existência de osteoporose por hipogonadismo. ${ }^{7}$

\section{Calcitonina}

No Brasil, a calcitonina de salmão foi aprovada para tratamento da osteoporose pós-menopausa na dose de 200 UI por dia nasal, com evidências da redução de fraturas vertebrais, sem ação documentada em osso cortical. A calcitocina tem efeito analgésico na dor da fratura vertebral e indicação para o tratamento na algoneurodistrofia ou atrofia de Sudeck, e está disponível para dispensação na rede SUS. O estudo Prevent Recurrence of Osteoporotic Fractures (PROOF, na sigla em inglês), no ano 2000, demonstrou eficácia em prevenção de fraturas vertebrais em osteoporose pós-menopausa, sem evidência de ação no osso cortical. É uma alternativa de segunda linha no tratamento da osteoporose, pois estudos de outros medicamentos mostraram melhores resultados em redução de fraturas. ${ }^{25,26}$

\section{Raloxifeno}

O raloxifeno, aprovado para tratamento de osteoporose pósmenopausa, está disponível para dispensação na rede SUS. É um medicamento do grupo dos SERM , os quais atuam de forma antagonista em alguns órgãos e agonista em outros, e possuem um efeito benéfico nos ossos. Indicado para prevenção e tratamento da osteoporose pós-menopausa, afetando mais osso trabecular (coluna vertebral), com redução significativa de fraturas vertebrais. Apresentação em cápsulas na dose de $60 \mathrm{mg}$ por dia. 0 raloxifeno é indicado também para redução de câncer de mama nas mulheres pós-menopausa com osteoporose. $\mathrm{O}$ medicamento não tem ação demonstrada em osso cortical, portanto sem eficácia demonstrada na prevenção de fraturas do quadril. Seu principal efeito colateral é o tromboembolismo venoso (TEV), e deve ser evitado em mulheres com história prévia e familiar de TEV. ${ }^{6,18,25}$

\section{Bisfosfonatos}

Bisfosfonatos são os medicamentos antirreabsortivos mais usados em todo o mundo para tratamento da osteoporose. ${ }^{15,16}$ São análogos sintéticos do pirofosfato que se ligam a hidroxiapatita no osso, inibindo a ação reabsortiva do osteoclasto. Devido à sua incorporação dentro do tecido ósseo durante o processo de remodelação, ${ }^{16}$ podem ser reciclados sob a superfície do osso, resultando em ação prolongada do medicamento. ${ }^{16}$ Os bisfosfonatos podem permanecer por até 10 
anos nos ossos, aqueles que possuem cadeia aminada, contendo nitrogênio, na molécula tem maior poder de ação.Os mais potentes são: zolendronato, risedronato, alendronato e ibandronato $^{17,18}$ São drogas de uso seguro, podendo causar hipocalcemia e dores musculares ocasionalmente. ${ }^{16}$

Dois eventos adversos sérios ocorrem raramente em uso prolongado: a fratura atípica do fêmur, na região subtrocanteriana, de traço transverso não cominutiva, com espessamento cortical lateral, podendo ser bilateral, ${ }^{16}$ e geralmente após 8 anos de uso contínuo. A osteonecrose mandibular é o outro evento, definida pela exposição óssea gengival maxilofacial. ${ }^{16}$ A osteonecrose mandibular ocorre mais em pessoas em tratamento de formas graves de câncer com um incidência de $1 / 10.000$ a $1 / 100.000$ pacientes por ano, sendo fatores de risco procedimentos dentários invasivos e pobre higiene bucal. ${ }^{19} \mathrm{O}$ site da Associação Brasileira de Ortopedia em Osteometabolismo (ABOOM) (https://www.aboom.com.br/) traz informações importantes sobre necrose de mandíbula, e também o FRAX Brasil. Deve-se evitar o uso em pacientes com clearance de creatinina $<35 \mathrm{ml}$ por minuto. ${ }^{16,18}$ Pessoas com baixo nível sérico de 25-hidroxivitamina-D desenvolvem hipocalcemia usando bisfosfonatos. $^{16}$

As doses diárias via oral de alendronato $10 \mathrm{mg}$ e risedronato $5 \mathrm{mg}$ são raramente usadas. 0 alendronato $70 \mathrm{mg}$ e o risedronato $35 \mathrm{mg}$ são de uso semanal (disponíveis nas farmácias do SUS). Uso de dose mensal para risedronato $150 \mathrm{mg}$ e ibandronato $150 \mathrm{mg}$. Bisfosfonatos orais são absorvidos de 1 a $5 \%$ no intestino, e desta quantidade, $50 \%$ liga-se ao osso, sendo o restante excretado pelos rins. ${ }^{18}$ Os comprimidos devem ser ingeridos com água pura, pela manhã em jejum, 60 minutos antes do café, e recomenda-se não se deitar para evitar possível refluxo gastroesofágico. No caso de intolerância gástrica, pode ser usado o ácido zolendrônico ou zolendronato, de $5 \mathrm{mg}$ em infusão venosa anual. ${ }^{18} \mathrm{O}$ principal efeito colateral dos injetáveis é a síndrome gripal (flu-like syndrome) devido à liberação de citocinas na fase aguda, causando febre e dores musculares. ${ }^{1,6,18}$ Este efeito diminui ou cessa continuando o tratamento. Os autores recomendam hidratar o paciente com 6 copos de líquidos por 3 dias antes e 3 dias após infusão, usar nesse período cálcio $500 \mathrm{mg} 3$ vezes ao dia, e 3 dias após, e acrescentar no dia da infusão acetaminofeno $500 \mathrm{mg}$ de $8 \mathrm{em} 8$ horas por até 3 dias, se necessário.

Não existe consenso definido de quanto tempo usar esses medicamentos; os bisfosfonatos não são exatamente iguais, portanto, deveriam existir estudos específicos individuais. Alendronato, risedronato e zolendronato são bem avaliados. Recomenda-se uso durante 3 anos do injetável (zolendronato) quando o paciente está em baixo risco para fraturas, e 5 anos do uso oral. ${ }^{6,16,20}$ Black et al $^{20}$ definem que pacientes com densidade mineral óssea baixa, T-Score $<-2.5$ no colo femoral, após 3 a 5 anos de tratamento, têm alto risco de fraturas vertebrais, bem como aqueles com fraturas vertebrais preexistentes mesmo com T-Score - 2.0 continuam sendo beneficiados com a continuidade do tratamento. Baixo risco são os pacientes com T-Score $>-2.0$, sem benefícios com tratamento continuado após 5 anos do oral ou 3 do injetável. ${ }^{20}$ Os dados de estudos dos bisfosfonatos para limite de uso são 10 anos; cada paciente deve ser avaliado com seus fatores de risco, efeitos colaterais e comorbidades existentes para tomada de decisão da parada ou continuidade do tratamento. Os bisfosfonatos são indicados também para homens. ${ }^{7,21}$

Bisfosfonatos podem ser utilizados em período agudo de fratura. ${ }^{22} \mathrm{O}$ ácido zolendrônico demonstrou aumento do calo ósseo em ratos. ${ }^{22}$ Há evidências para suspender uso de bisfosfonatos em fraturas de pacientes que estão em uso prolongado, e em casos de fratura atípica. ${ }^{22}$ Em um estudo de fraturas do punho, o tratamento conservador demonstrou evitar perda na densidade mineral pela imobilização, com o uso de risedronato, e não demonstrou superioridade no tempo de consolidação da fratura em relação a placebo (uso de cálcio e vitamina D). ${ }^{23}$

\section{Denosumabe}

O denosumabe é o primeiro tratamento biológico aprovado para o tratamento da osteoporose, feminina e masculina. ${ }^{6,7,16}$ Ele inibe a reabsorção óssea ligando ao RANKL, do grupo do fator ativador de necrose tumoral, diminuindo a diferenciação dos osteoclastos (TNF). ${ }^{16} \mathrm{O}$ denosumabe pode ser utilizado em pacientes com comprometimento renal. $\mathrm{O}$ medicamento é apresentado de forma injetável subcutânea com $60 \mathrm{mg} / \mathrm{ml}$, dose semestral, seringas prontas para uso com $1 \mathrm{ml}$. É um biológico similar da osteoprotegerina, molécula que inibe a formação dos osteoclastos, bloqueando o RANKL, que ativa o RANK para formação da célula gigante multinuclear - osteoclasto, responsável pela reabsorção óssea. O denosumabe é indicado para tratamento de mulheres na pós-menopausa e de pacientes com intolerância aos bifosfonatos. ${ }^{6}$ Também é indicado para uso em homens.

Diferentemente dos bisfosfonatos, o denosumabe pode ser utilizado em pacientes com função renal comprometida. ${ }^{1,6,16}$ Um grande ensaio envolvendo mulheres com TScore em densitometria entre - 2.5 e - 4.0 na coluna lombar ou fêmur total em tratamento com denosumabe mostrou diminuição de $68 \%$ na incidência das fraturas vertebrais, de $40 \%$ de fraturas do fêmur e de $20 \%$ nas não vertebrais em relação à população placebo. ${ }^{1,16}$ Raros casos de fraturas atípicas do fêmur e osteonecrose de mandíbula foram relatados. $^{1,16}$ Dados de 10 anos de tratamento mostraram ganhos contínuos da DMO sem limite com o tempo de uso, redução sustentada de fraturas e bom perfil de segurança, mesmo com disfunção renal. ${ }^{2,6}$ A descontinuação do tratamento com denosumabe pode levar à reversão dos ganhos obtidos, verificado em exames de densitometria óssea no seguimento clínico. Caso isso ocorra, deve ser feita troca por outro medicamento. ${ }^{6,16,24}$ O denosumabe também é aprovado para uso em homens e na osteoporose induzida por corticoides.

\section{Anabólico Ósseo}

Os osteoanabólicos são indicados em quatro grupos de paciente: 1 - osteoporose grave com fraturas, ou alto risco de fraturas; 2 - insuficiência de tratamento, mantendo baixa DMO ou ocorrência de fraturas; 3 - intolerância ou 
contraindicações para outras alternativas de tratamento da osteoporose; 4- osteoporose induzida por corticóides. 27

\section{Teriparatida}

A teriparatida é um análogo do PTH com sequência de 34 aminoácidos (PTH 1-34), e estimula o metabolismo ósseo com predomínio da formação, aumentando a massa óssea trabecular e cortical. O medicamento é administrado em injeções subcutâneas diárias de $20 \mu \mathrm{cg} .{ }^{2,6,28}$ A teriparatida apresentou redução de risco de fraturas na coluna vertebral de $65 \%$, e em fraturas não vertebrais de $54 \%{ }^{28}$ Não foi demonstrado na amostra estuda número siginificativo de redução de fraturas do quadril, ${ }^{28}$ talvez devido ao número da amostra, mas na prática clínica seu uso tem demonstrado proteção. O período de tratamento foi limitado a 2 anos devido ao aparecimento de osteossarcoma em ratos. ${ }^{28}$

Há grande ganho de massa óssea mais evidente em osso trabecular (coluna vertebral). Para a manutenção dos ganhos, é utilizado no seguimento um antirreabsortivo. A teriparatida é um medicamento seguro para uso. Pode ocasionar hipercalcemia assintomática, náusea ocasional, tontura, câimbras ou cefaleia. ${ }^{6,18}$ A teriparatida está contraindicada para uso em pacientes com alto risco de osteossarcoma, tais como crianças e adolescentes, doença de Paget, metástases ósseas, pós-radioterapia do esqueleto, e ou aumentos não explicados

\section{FLUXOGRAMA DE TRATAMENTO MEDICAMENTOSO DA OSTEOPOROSE PRIMÁRIA}

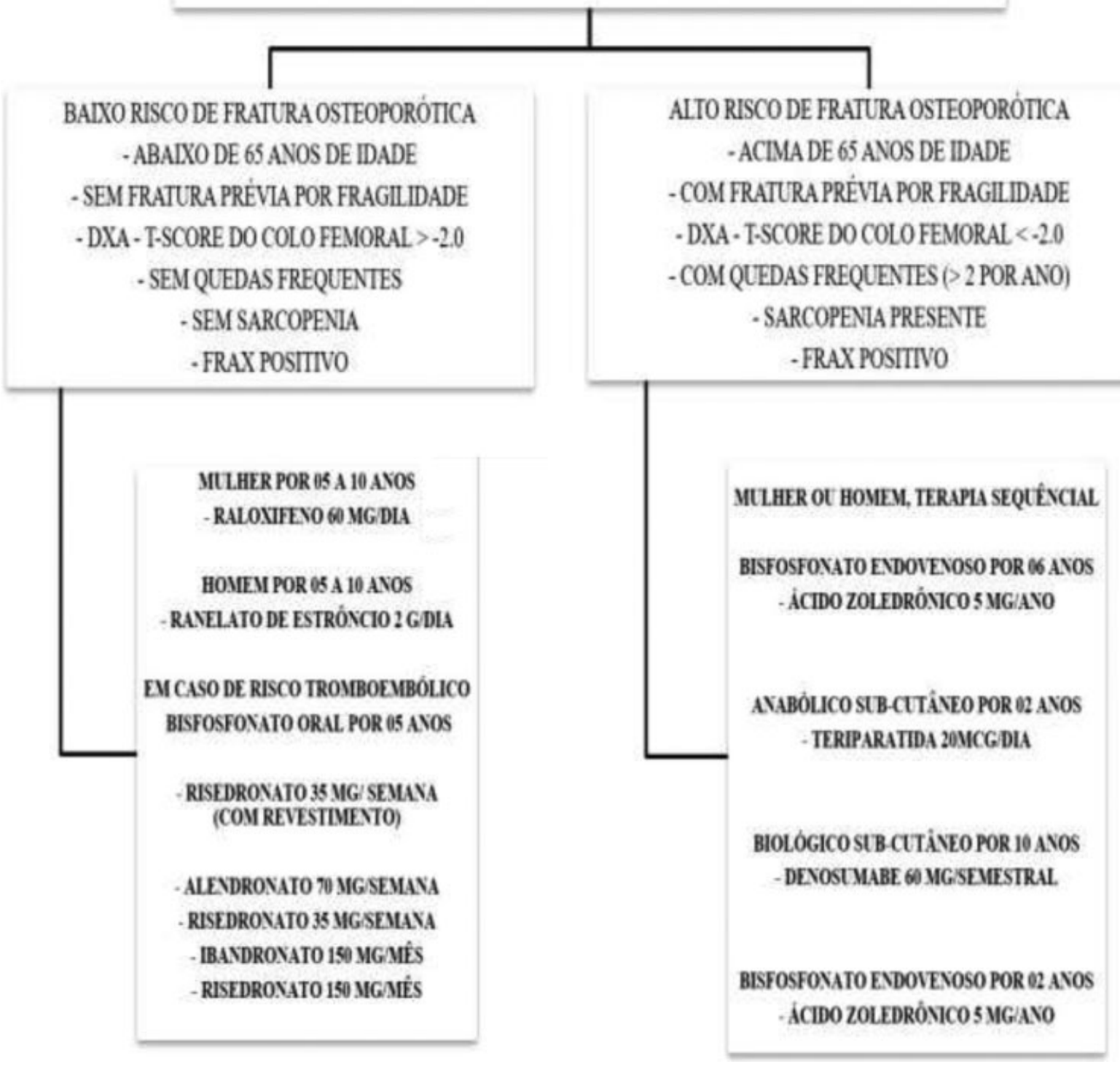

Fig. 1 Fluxograma de tratamento medicamentoso da osteoporose primária. 
de fosfatase alcalina. ${ }^{6,18,27}$ Por prudência, evita-se o uso em qualquer tipo de câncer diagnosticado, e em hiperparatireoidismo primário e secundário. Na deficiência de vitamina D, pode ocorrer hiperparatireoidismo secundário, corrigido com reposição de vitamina $\mathrm{D}$, podendo neste caso utilizar teriparatida. A teriparatida tem indicação para tratamento de osteoporose grave induzida por corticóides, tanto em mulheres como em homens. ${ }^{29}$ Um estudo comparativo com bisfosfonatos demonstrou melhora na consolidação de fraturas vertebrais com teriparatida. ${ }^{29}$

\section{Medicamentos Anabólicos Ainda Não Aprovados Pela Anvisa}

\section{Abaloparatida}

Abaloparatida é um análogo do PTHrP humano ${ }^{1-3334}$ desenvolvido para tratamento da osteoporose. A sequência de aminoácidos é idêntica à da proteína relacionada ao hormônio da paratireóide (PTHrP) nos primeiros 20, enquanto os remanescentes são diferentes. ${ }^{31}$ No estudo Abaloparatide Comparator Trial in Vertebral Endpoints (ACTIVE, sigla em inglês), com abaloparatida $80 \mu$ gr subcutâneo diário por 18 meses, reduziu o risco de novas fraturas vertebrais em $86 \%$ e de fraturas não vertebrais em $43 \%$, e também reduziu em $70 \%$ fraturas osteoporóticas maiores (clínicas vertebrais, úmero proximal e quadril) comparados com placebo. ${ }^{32} \mathrm{O}$ medicamento tem o potencial de reduzir a incidência de fraturas vertebrais e não vertebrais, e de aumentar a DMO em mulheres com osteoporose pós-menopausa, independente da idade, história de fratura anterior ou DMO baixa. ${ }^{32}$ Nas fraturas vertebrais, não foi encontrada diferença significativa entre abaloparatida e teriparatida. ${ }^{27}$ Indicado para pacientes com alto risco de fraturas, insucesso com tratamentos anteriores e intolerância aos medicamentos usuais. $\mathrm{O}$ uso de abaloparatida e de teriparatida são restritos por até 2 anos. ${ }^{27} \mathrm{~A}$ abaloparatidda possui as mesmas contraindicações da teriparatida, e é aprovada nos Estados Unidos, Europa e outros países.

\section{Romosozumabe}

Romosozumabe foi desenvolvido após estudo da doença rara, genética, com alta massa óssea, a esclerosteose, devido à mutação com perda funcional no gene esclerostina (SOST). A esclerostina é secretada pelos osteócitos e inibe a formação e estimula a reabsorção óssea. Romosozumabe é um anticorpo monoclonal humanizado anti-esclerostina. ${ }^{27}$ No estudo Fracture Study in Postmenopausal Women with Osteoporosis (FRAME), com injeção subcutânea mensal de $210 \mathrm{mg}$ durante 12 meses, houve redução de $75 \%$ na incidência de novas fraturas vertebrais comparado com placebo. ${ }^{27} \mathrm{O}$ aumento da DMO foi mais rápido e maior do que com placebo, alendronato, denosumabe e teriparatida, na coluna vertebral e no quadril. $^{33}$

Para otimizar a utilização dos medicamentos conforme o risco de fratura, sugerimos o fluxograma abaixo (-Figura 1).

\section{Considerações Finais}

Assim, consideramos que a osteoporose é uma doença crônicodegenerativa do esqueleto, de extrema importância, pois está se desenvolvendo em uma pandemia, devido ao envelhecimento populacional. Sua consequência é a fratura por fragilidade, que aumenta a morbimortalidade, principalmente nas fraturas do quadril e da coluna. Na ortopedia e traumatologia mundial, existe uma falta de conhecimento sobre a doença, o que leva à diminuição do diagnóstico e do tratamento não cirúrgico e medicamentoso, com foco na prevenção secundária de fraturas. No presente artigo, demonstramos as várias opções de tratamento disponíveis com suas indicações, eventos adversos e sugestões de fluxograma de tratamento. O ortopedista e traumatologista deve reconhecer a gravidade dessa doença e suas consequências, e se não se sentir habilitado para esse tratamento, deve encaminhar o paciente para colegas que lidam com essa situação.

\section{Suporte Financeiro}

Não houve suporte financeiro de fontes públicas, comerciais, ou sem fins lucrativos.

Conflito de Interesses

Os autores declaram não haver conflito de interesses.

\section{Referências}

1 Black DM, Rosen CJ. Clinical Practice. Postmenopausal Osteoporosis. N Engl J Med 2016;374(03):254-262

2 de Souza MP. Osteoporosis Diagnosis and Treatment. Rev Bras Ortop 2010;45(03):220-229

3 Stolnick B, Oliveira LG. Para que a primeira fratura seja a última. Rev Bras Ortop 2016;51(02):121-126

4 Freedman BA, Potter BK, Nesti LJ, Cho T, Kuklo TR. Missed opportunities in patients with osteoporosis and distal radius fractures. Clin Orthop Relat Res 2007;454(454):202-206

5 Souza BGSE, Carvalho LGVA, Oliveira LFMM, Ferreira AG, Amaral RCSD, Oliveira VM. Primary and secondary osteoporotic fractures prophylaxis: evaluation of a prospective cohort. Rev Bras Ortop 2017;52(05):538-543

6 Radominski SC, Bernardo W, Paula AP, Albergaria BH, Moreira C, Fernandes CE. Diretrizes brasileiras para o diagnóstico e tratamento da osteoporose em mulheres na pós-menopausa. Rev Bras Reumatol 2017;57(Suppl 2):s452-s466

7 Oliveira LG, Guimarães ML. Male osteoporosis. Rev Bras Ortop 2015;45(05):392-396

8 Banefelt J, Åkesson KE, Spångéus A, et al. Risk of imminent fracture following a previous fracture in a Swedish database study. Osteoporos Int 2019;30(03):601-609

9 Sousa CJ, Oliveira ML. Ferramenta FRAX no Brasil: revisão integrativa da literatura após sua Validação. Rev Bras Geriatr Gerontol 2018;21(01):111-118

10 Bauer DC. Clinical practice. Calcium supplements and fracture prevention. N Engl J Med 2013;369(16):1537-1543

11 Peters BS, Martini LA. Nutritional aspects of the prevention and treatment of osteoporosis. Arq Bras Endocrinol Metabol 2010;54 (02):179-185

12 Chen LR, Wen YT, Kuo CL, Chen KH. Calcium and Vitamin D Supplementation on Bone Health: Current Evidence and Recommendations. Int J Gerontol 2014;8:183-188 
13 Kanis JA, Cooper C, Rizzoli R, Reginster JY; Scientific Advisory Board of the European Society for Clinical and Economic Aspects of Osteoporosis (ESCEO) and the Committees of Scientific Advisors and National Societies of the International Osteoporosis Foundation (IOF). European guidance for the diagnosis and management of osteoporosis in postmenopausal women. Osteoporos Int 2019;30(01):3-44

14 Maeda SS, Borba VZ, Camargo MB, et al, Brazilian Society of Endocrinology and Metabology (SBEM). Recommendations of the Brazilian Society of Endocrinology and Metabology (SBEM) for the diagnosis and treatment of hypovitaminosis D. Arq Bras Endocrinol Metabol 2014;58(05):411-433

15 Qaseem A, Forciea MA, McLean RM, Denberg TD; Clinical Guidelines Committee of the American College of Physicians. Treatment of Low Bone Density or Osteoporosis to Prevent Fractures in Men and Women: A Clinical Practice Guideline Update From the American College of Physicians. Ann Intern Med 2017;166(11):818-839

16 Matzkin EG, DeMaio M, Charles JF, Franklin CC. Diagnosis and Treatment of Osteoporosis: What Orthopaedic Surgeons Need to Know. J Am Acad Orthop Surg 2019;27(20):e902-e912

17 Watts NB, Diab DL. Long-term use of bisphosphonates in osteoporosis. J Clin Endocrinol Metab 2010;95(04):1555-1565

18 Maeda SS, Lazaretti-Castro M. An overview on the treatment of postmenopausal osteoporosis. Arq Bras Endocrinol Metabol 2014;58(02):162-171

19 Khan AA, Morrison A, Hanley DA, et al, International Task Force on Osteonecrosis of the Jaw. Diagnosis and management of osteonecrosis of the jaw: a systematic review and international consensus. J Bone Miner Res 2015;30(01):3-23

20 Black DM, Bauer DC, Schwartz AV, Cummings SR, Rosen CJ. Continuing bisphosphonate treatment for osteoporosis-for whom and for how long? N Engl J Med 2012;366(22):2051-2053

21 Zanatta LB, Marcattoa C, Ramosa CS, Manasa N, Moreira C, Borba V. Uso de pamidronato para tratamento da osteoporose no sistema público de saúde no Brasil. Rev Bras Reumatol 2017;57(06):514-520

22 Kates SL, Ackert-Bicknell CL. How do bisphosphonates affect fracture healing? Injury 2016;47(01 Suppl 1):S65-S68

23 Oliveira LG, Eis SR, Neto HM, de Moraes FB, Pires LA, Vasconcelos JW. Use of risedronate for consolidation and callus formation in
Colles fractures in postmenopausal women: SOLID study. Rev Bras Ortop 2015;50(03):274-282

24 Cummings SR, Ferrari S, Eastell R, et al. Vertebral Fractures After Discontinuation of Denosumab: A Post Hoc Analysis of the Randomized Placebo-Controlled FREEDOM Trial and Its Extension. J Bone Miner Res 2018;33(02):190-198

25 Protocolos clínicos e diretrizes terapêuticas: volume 3 / Ministério da Saúde, Secretaria de Atenção à Saúde. Brasília: Ministério da Saúde; 2014. Disponível em: http://portalsaude.saude.gov.br/index.php/o-ministerio/principal/leia-mais-oministerio/840sctie-raiz/daf-raiz/cgceaf-raiz/cgceaf/13-cgceaf/11646-pcdt

26 Chesnut CH III, Silverman S, Andriano K, et al, PROOF Study Group. A randomized trial of nasal spray salmon calcitonin in postmenopausal women with established osteoporosis: the prevent recurrence of osteoporotic fractures study. Am J Med 2000;109 (04):267-276

27 Haas AV, LeBoff MS. Osteoanabolic Agents for Osteoporosis. J Endocr Soc 2018;2(08):922-932

28 Neer RM, Arnaud CD, Zanchetta JR, et al. Effect of parathyroid hormone (1-34) on fractures and bone mineral density in postmenopausal women with osteoporosis. N Engl J Med 2001;344 (19):1434-1441

29 Pereira RM, Carvalho JF, Paula AP, et al. Diretrizes para prevenção e tratamento da osteoporose induzida por glicocorticoide. Rev Bras Reumatol 2012;52(04):569-593

30 Iwata A, Kanayama M, Oha F, Hashimoto T, Iwasaki N. Effect of teriparatide (rh-PTH 1-34) versus bisphosphonate on the healing of osteoporotic vertebral compression fracture: A retrospective comparative study. BMC Musculoskelet Disord 2017;18(01):148

31 Gonnelli S, Caffarelli C. Abaloparatide. Clin Cases Miner Bone Metab 2016;13(02):106-109

32 Cosman F, Hattersley G, Hu MY, Williams GC, Fitzpatrick LA, Black DM. Effects of Abaloparatide-SC on Fractures and Bone Mineral Density in Subgroups of Postmenopausal Women With Osteoporosis and Varying Baseline Risk Factors. J Bone Miner Res 2017;32 (01):17-23

33 McClung MR, Grauer A, Boonen S, et al. Romosozumab in postmenopausal women with low bone mineral density. N Engl J Med 2014;370(05):412-420 